NASA Technical Memorandum 102619

\title{
INTEGRATED CONTROL/STRUCTURE OPTIMIZATION BY MULTILEVEL DECOMPOSITION
}

Thomas A. Zeiler and Michael G. Gilbert

\section{March 1990}

(NASA-TM-102G19) INTEGRATEO

CONTROL/STRUCTURE OPTIMIZATION BY MULTILEVEL

OECOMPUSITION (NASA) $13 \mathrm{p}$
N90-19281

CSCL 228
Unclas

0271140

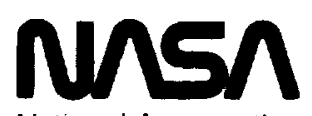

National Aeronautics and

Space Administration

Langley Research Center

Hampton, Virginia 23665-5225 
$+\cdots$

$=\cdots$ 


\section{Integrated Control/Structure Optimization by Multilevel Decomposition}

\author{
Thomas A. Zeiler* \\ Lockheed Engineering and Sciences Co. \\ Hampton, Va. 23665 \\ and
}

\begin{abstract}
A method for integrated control/structure optimization by multilevel decomposition is presented. It is shown that several previously reported methods were actually partial decompositions wherein only the control was decomposed into a subsystem design. One of these partially decomposed problems was selected as a benchmark example for comparison. The present paper fully decomposes the system into structural and control subsystem designs and produces an improved design. Theory, implementation, and results for the method are presented and compared with the benchmark example.
\end{abstract}

\section{INTRODUCTION}

Over the last decade, increasing attention has been given to control/structure interaction (CSI) problems and the integrated design of a structure and its controller. In general, the approaches to integrated design can be categorized as either sequential or simultaneous [1]. A sequential method is one in which one disciplinary design iteration precedes the other. The interactions between the structure and control are examined in an analysis step but are not rigorously included in the determination of design changes in the next iteration step. A simultaneous design method is one in which the control and structure design problems are combined into a single design problem. Interactions are considered at the outset and the effects of the structural and control design variables are considered together. References 1 through 19 give examples of simultaneous structure and control design approaches, each of them optimization-based.

Study of these papers shows that there are a variety of possible approaches to the problem of simultaneous structure and control design even within the limits of being based in optimization

\footnotetext{
* Staff Engineer, Member, AIAA

** Aerospace Engineer, Senior Member, AJAA
}

\author{
Michael G. Gilbert** \\ NASA Langley Research Center \\ Hampton, Va. 23665
}

methods. Often, the objective function is structural weight plus some weighted function of controlled response. Typical constraints might be on closedloop frequencies or structural deflections. Usually, the methods proposed have stated the design problem as a single optimization problem wherein detailed structural design variables (member sizes) and control gains are of equal status as problem design variables. The problems on which these methods have been exercised have been relatively small, consisting of a simple structure with a few structural design variables and simple controllers with a few gains. While these methods have worked well, they could easily require extremely large design problems for a large, complex structure. To make the problem size more manageable, the number of design variables might have to be reduced to ineffectual levels. There is also an organizational problem associated with combining the synthesis of structure and controller so directly. A typical aerospace design organization is highly segregated along disciplinary lines. Obviously, sufficient communication exists between disciplinary design groups to effect the design of successful aerospace syslems. However, there are cases of actual flight hardware with problems that might well have been caught before production had the design process more closely coordinated structure and control design functions.

Multilevel decomposition is an alternative approach to large multidisciplinary system design that has been proposed [20]. In this approach, a large multidisciplinary system is broken down along disciplinary and hierarchial lines into subsystem designs that are smaller and more easily managed than the complete, integrated system. The subsystem designs are coordinated at a higher level where the influences from each subsystem are integrated. At this top level, the design variables quantify the influence of the subsystems on the total system, but are not at the level of detailed design. Desired changes in these variable are passed to the appropriate subsystem design functions wherein detailed designs are accomplished treating the quantities specified from above as parameters to be held constant. An important element of this process is the return, from the subsystem designs to the upper level, of the values of sensitivity derivatives with respect to the parameters held 
constant. These sensitivities are used at the top level to construct gradients for use in an optimization. The sensitivities may be of any quantities that influence the objective and constraint functions being used at the top level. If the subsystem designs are themselves accomplished through optimization, then the sensitivities would be the so-called "optimum sensitivity derivatives" [21] or, more correctly, "sensitivities of the optimum". Thus these sensitivities account for how the optimum subsystem design will change as the parameters are changed. Further, since the controlling influence upon the disciplinary designs exists at the top level where the design variables are related to each of the contributing subsystems, the top level design is a simultaneous one.

Several of the simultaneous structure and control design methodologies cited in this paper $[5$, 13, 15-17] have actually been set, knowingly or not, in this multilevel format. In these papers, the structure was represented completely at the top level with no decomposition. The structural design variables were either the detailed structural sizes or more global structural parameters (such as stiffness characteristics) without regard to the detailed structural design. All of them, however, also incorporated the optimal, steady-state, Linear Quadratic Regulator (LQR) as controller. At each iteration step, a LQR design is obtained and sensitivities of this optimum are computed with respect to the top level variables (structural sizes and, in some cases, elements of the weighting matrices used in the LQR synthesis), and used at the top level in constructing gradients of the objective and constraint functions for the next iteration. Sensitivity equations for the LQR and LQG (Linear Quadratic Gaussian for systems incorporating Gaussian white noise) control have been well developed over the past years [22]. Sensitivities and even multilevel decompositions for purely structural systems under static loading have also been developed [23, 24]. However, to date, decomposition of both structure and control in the controlled structure design problem has not been achieved. It is the purpose of this paper to report on a simultaneous structure and control optimization wherein the total system has been decomposed into both structural and control subsystems.

\section{MODEL DESCRIPTION}

The structural model chosen for this study is the ACOSS-4 [25], shown in figure 1. The tetrahedral truss, mounted on three bipods, is intended to model a feed tower for a class of large space antennae. Colinear with each bar in the bipods, an axial force actuator is assumed to exist. All geometric and material properties are assumed to be in unspecified but consistent units. Table I gives the nodal coordinates, and Table II gives the nodal connectivities and the initial cross-sectional areas of the truss elements. The elastic modulus of each member is 1.0 and the mass density is 0.001 . A concentrated mass of 2.0 is assumed to be at each of the four comers. Further, the mass "units" are assumed to be such that the total mass of the structural elements is negligible in comparison to the total of the concentrated masses. Thus the structural mass does not enter into the dynamic characteristics of the model. This model is a good compromise between simplicity and complexity, and is well represented in the open literature. The controller is chosen to be the optimal steady-state LQR. Its use will also allow comparisons to be made with published results [17].

\section{PROBLEM}

\section{Equations of Motion and LQR Control}

The matrix equations of undamped, forced motion for the finite element model of the structure take the form,

$$
\mathbf{M} \ddot{\mathbf{w}}+\mathbf{K} \mathbf{w}=\mathbf{b u}
$$

where $\mathbf{M}$ and $\mathbf{K}$ are the mass and stiffness matrices, and $w$ is the vector of $x, y$, and $z$ displacements of each of the comer nodes. The matrix $b$ is the input matrix defining the geometry of the applied load distribution. In this case it contains the direction cosines of the six actuators. The input vector $u$, then, is composed of the forces exerted by each of the actuators. The undamped natural modes of the structure are obtained by solving the eigenvalue problem,

$$
\left[\mathbf{K}-\omega_{i}^{2} \mathbf{M}\right] \boldsymbol{\phi}_{i}=\mathbf{0}
$$

Assuming linearly independent eigenvectors, the damped equations of motion can then be cast in modal form,

$$
\overline{\mathbf{M}} \ddot{\mathbf{x}}+2 \Omega \zeta \dot{\mathbf{x}}+\overline{\mathbf{K}} \mathbf{x}=\overline{\mathbf{b}} \mathbf{u} .
$$

where

and

$$
\begin{gathered}
\overline{\mathbf{M}}=\Phi^{\mathrm{T}} \mathbf{M} \Phi=\mathrm{I} \\
\overline{\mathbf{K}}=\Phi^{\mathrm{T}} \mathrm{K} \Phi=\Omega^{2} \\
\overline{\mathbf{b}}=\Phi^{\mathrm{T}} \mathrm{b}
\end{gathered}
$$

$$
\mathbf{w}=\Phi \mathbf{x}
$$


Above, $\zeta$ is a diagonal matrix of modal damping factors, $\Phi$ is the modal matrix containing the eigenvectors obtained in the solution of equation 2 , $\Omega^{2}$ is a diagonal matrix of squared structural frequencies from the solution of equation $2, x$ is the vector of generalized coordinates, and $I$ is an identity matrix.

The equations of motion may then be put in state-space form,

$$
\dot{\mathbf{X}}=\mathbf{A X}+\mathbf{B u}
$$

where

$$
\begin{gathered}
X=\left\{\begin{array}{l}
x \\
\dot{x}
\end{array}\right\} \\
A=\left[\begin{array}{cc}
0 & I \\
-\Omega^{2} & -2 \Omega \zeta
\end{array}\right]
\end{gathered}
$$

and

$$
\mathbf{B}=\left[\begin{array}{l}
0 \\
\bar{b}
\end{array}\right]
$$

A linear state feedback control law would be of the form,

$$
\mathbf{u}=-\mathbf{G X}
$$

The LQR control law is that which minimizes the cost function,

$$
J=\int_{0}^{\bar{T}}\left\{\mathbf{X}^{\mathrm{T}} \mathbf{Q X}+\mathbf{u}^{\mathrm{T}} \mathbf{R} \mathbf{u}\right\} \mathrm{dt}
$$

subject to the constraint of equation 4. The matrices $\mathbf{Q}$ and $\mathbf{R}$ are weighting matrices. The gain matrix is found from,

$$
\mathbf{G}=\mathbf{R}^{-1} \mathbf{B}^{\mathrm{T}} \mathbf{P}
$$

where the symmetric matrix $P$ is the minimum solution to the steady-state, algebraic matrix Riccati equation,

$$
\mathbf{A}^{\mathrm{T}} \mathbf{P}+\mathbf{P A}-\mathbf{P B R}^{-1} \mathbf{B}^{\mathrm{T}} \mathbf{P}+\mathbf{Q}=\mathbf{0}
$$

Then, the equations of motion of the closed-loop system may be written,

$$
\dot{\mathbf{x}}=\mathbf{A}_{\mathrm{cl}} \mathbf{X}
$$

where

$$
A_{d}=A \cdot B G
$$

\section{Benchmark Example}

The design problem to be considered, taken from reference 17 , is the minimization of the Frobenius norm of the feedback gain matrix, subject to lower bound (lb) constraints on the first two closed-loop (cl) frequencies and the damping parameter of the first closed-loop mode. Side constraints are imposed on the cross-sectional areas of the truss members, which are the design variables, y. Also, the open-loop damping factors, $\zeta$, are assumed to be zero. The problem reads:

$$
\min _{y} F r=\operatorname{Tr}\left(G^{T} R_{G} G\right)
$$

subject to:

$$
\begin{gathered}
\omega_{\mathrm{cl}_{1}} \geq \omega_{\mathrm{cl}_{1}}^{\mathrm{bb}} \\
\omega_{\mathrm{cl}_{2}} \geq \omega_{\mathrm{cl}_{2}}^{\mathrm{b}} \\
\xi_{\mathrm{cl}_{1}} \geq \xi_{\mathrm{cl}_{1}}^{\mathrm{b}} \\
y_{\mathrm{lb}} \leq \mathrm{y} \leq \mathrm{y}_{\mathrm{ub}} \quad \text { for each } \mathrm{y}
\end{gathered}
$$

where, for eigenvalue

$$
\lambda=\sigma \pm j \omega
$$

the damping parameter is defined,

$$
\xi=\frac{-\sigma}{\sqrt{\sigma^{2}+\omega^{2}}}
$$

The gain weighting matrix, $\mathbf{R}_{\mathrm{G}}$, is taken to be an identity matrix and is distinct from the control weighting matrix in equation 6 . The weighting matrices $\mathbf{Q}$ and $\mathbf{R}$ in equation 6 are also identity matrices. In reference 17 , the lower bounds in constraint equations $10 \mathrm{~b}-\mathrm{d}$ are $1.341 \mathrm{r} / \mathrm{s}, 1.6 \mathrm{r} / \mathrm{s}$ and 0.15 , respectively. The lower bound on each of the design variables was 10 while two different upper bounds (ub) were considered. One was 1000 , and is referred to as 'Design II' and the other was 2000, referred to as 'Design III'.

A description of the method used in reference 17 will be given partly because similar ideas are important for the present formulation. It will also facilitate demonstrating how the approach of reference 17 (as well as references 5, 13, 15, and 16) was actually a partial decomposition. Some results from reference 17 will be shown later for comparison with the present results. Gradients of the Frobenius gain norm and the constraints are found from,

$$
\begin{gathered}
\frac{\partial F_{r}}{\partial y}=\operatorname{Tr}\left(\frac{\partial G^{T}}{\partial y} R_{G} G+G^{T} R_{G} \frac{\partial G}{\partial y}\right) \\
\frac{\partial \omega_{c_{i}}}{\partial y}=\operatorname{Im}\left(\frac{\partial \lambda_{c_{i}}}{\partial y}\right)
\end{gathered}
$$




$$
\frac{\partial \xi_{\mathrm{cl}_{i}}}{\partial y}=\frac{\omega_{\mathrm{cl}_{i}}}{\left(\sigma_{c_{i}}^{2}+\omega_{c_{i}}^{2}\right)^{3 / 2}}\left(\sigma_{\mathrm{ci}_{i}} \frac{\partial \omega_{\mathrm{cl}_{i}}}{\partial y}-\omega_{c_{i}} \frac{\partial \sigma_{c_{i}}}{\partial y}\right)
$$

where

$$
\frac{\partial \sigma_{c_{i}}}{\partial y}=\operatorname{Re}\left(\frac{\partial \lambda_{c_{i}}}{\partial y}\right)
$$

and

$$
\frac{\partial \lambda_{\mathrm{cl}_{\mathrm{i}}}}{\partial \mathrm{y}}=\Psi_{\mathrm{L}_{\mathrm{i}}}^{\mathbf{T}}\left(\frac{\partial \mathrm{A}}{\partial \mathrm{y}} \cdot \frac{\partial \mathrm{B}}{\partial \mathrm{y}} \mathbf{G} \cdot \mathbf{B} \frac{\partial \mathrm{G}}{\partial \mathrm{y}}\right) \Psi_{\mathrm{R}_{\mathrm{i}}}
$$

Since the eigenvalues occur in complex conjugate pairs, care must be taken when evaluating equation 12 that the derivatives have the proper signs. Above, $\psi_{L}$ and $\psi_{R}$, are left and right eigenvectors for the eigenvalue problem,

$$
\text { [I } \left.\lambda_{i}-\mathbf{A}\right] \psi_{i}=0
$$

The derivatives of the $\mathbf{A}$ and $\mathbf{B}$ matrices are,

$$
\begin{gathered}
\frac{\partial A}{\partial y}=\left[\begin{array}{cc}
0 & 0 \\
-\frac{\partial \Omega^{2}}{\partial y} & -2 \frac{\partial \Omega}{\partial y} \zeta
\end{array}\right] \\
\frac{\partial B}{\partial y}=\left[\begin{array}{cc}
0 \\
\frac{\partial \Phi^{T}}{\partial y} & b
\end{array}\right]
\end{gathered}
$$

where each diagonal element of the derivative of the $\Omega^{2}$ matrix is,

$$
\frac{\partial \omega_{j}^{2}}{\partial y}=\phi_{L_{j}} r\left(\frac{\partial K}{\partial y}-\omega_{j}^{2} \frac{\partial M}{\partial y}\right) \phi_{R_{j}}
$$

In the interests of brevity, the expressions for the derivatives in equation 16 of the eigenvectors will not be reproduced here as they are given in reference 17.

Finally, the key element is the derivative of the feedback gain matrix,

$$
\frac{\partial G}{\partial y}=R^{-1} \frac{\partial B^{T}}{\partial y} \mathbf{P}+\mathbf{R}^{-1} \mathbf{B}^{T} \frac{\partial \mathbf{P}}{\partial \mathbf{y}}
$$

The required derivative of the Riccati solution, $P$, is obtained by differentiating the Riccati equation to yield,

$$
\begin{aligned}
& \frac{\partial \mathbf{P}}{\partial y}\left(\mathbf{A} \cdot \mathbf{B R}^{-1} \mathbf{B}^{\mathrm{T}} \mathbf{P}\right)+\left(\mathbf{A} \cdot \mathbf{B R}^{-1} \mathbf{B}^{T} \mathbf{P}\right)^{\mathrm{T}} \frac{\partial \mathbf{P}}{\partial y} \\
& +\left[\mathbf{P} \frac{\partial A}{\partial y}+\frac{\partial A^{T}}{\partial y} \mathbf{P} \cdot \mathbf{P}\left(\frac{\partial B}{\partial y} R^{-1} B^{T}+B R^{-1} \frac{\partial B^{T}}{\partial y}\right) \mathbf{P}\right]=0
\end{aligned}
$$

This equation is a Lyapunov equation with $\partial \mathbf{P} / \partial \mathbf{y}$ as the unknown. Since the LQR control solution guarantees an asymtotically stable closed-loop system, the coefficient matrices (which are $A_{c l}$ and $\boldsymbol{A}_{\mathrm{cl}}{ }^{\mathrm{T}}$ ) of $\partial \mathbf{P} / \partial \mathrm{y}$ are asymtotically stable. Thus an unique solution for $\partial \mathbf{P} / \partial \mathbf{y}$ is guaranteed [22].

As mentioned earlier, this organization of the design problem is a partial multilevel decomposition, as shown in figure 2 , wherein only the control design is decomposed into an optimal subsystem design. The determination of the detailed structural design variables is handled at the top level and there is no structural decomposition. The structural design variables serve as constant parameters for the control design and sensitivities of the optimal control solution, and resulting behavior variables (closed-loop eigenvalues), are computed. As described in reference 21 , the optimum sensitivities are obtained through differentiation of the conditions of optimality satisfied by the solution of the optimization problem. The Riccati equation (equation 8) fills the role of optimality conditions for the optimal steady-state LQR problem.

\section{Present Formulation - General}

A generalized decomposition would appear as shown in figure 3 wherein the objective function is minimized with respect to higher level design variables, denoted by $z$ 's, that characterize subsystems I and II. In each iteration, the subsystem optimizations are performed with equality constraints enforcing the invariance of the parameters, $z$, specified as design variables at the higher level. After each subsystem optimization, sensitivities of the optimum solutions are computed, as are sensitivities of appropriate behavior variables, denoted in figure 3 by q's, that may be of importance to the top level design. The behavior variables might be used in the top level objective or constraint functions. In the development that follows, optimum sensitivities are indicated by superscript asterisks.

The general top level optimization problem is given by,

$$
\min _{\mathbf{z}} F(z, q)
$$

subject to:

$$
\begin{aligned}
& \mathrm{g}_{\mathrm{i}}(\mathrm{z}, q) \leq 0 \\
& \mathrm{z}_{\mathrm{b}} \leq \mathrm{z} \leq \mathrm{z}_{\mathrm{ub}}
\end{aligned}
$$

The optimization problem for subsystem I would be,

$$
\min _{y_{I}} F_{I}\left(y_{I}, q_{I}\left(y_{I}\right), q_{I I}\left(y_{I I}\right)\right)
$$


subject to:

$$
\begin{gathered}
g_{I_{i}}\left(y_{I}, q_{I}\left(y_{I}\right), q_{I I}\left(y_{I I}\right)\right) \leq 0 \\
z_{1}=z_{I}\left(y_{1}, q_{I}\left(y_{I}\right), q_{I I}\left(y_{I I}\right)\right) \\
y_{I_{I b}} \leq y_{I} \leq y_{I_{u b}}
\end{gathered}
$$

Note that dependencies upon behavior variables, $q_{n}$, from subsystem II are included in the functional statements above. This is because there may be strong physical coupling between the two subsystems. If so, then a certain amount of iteration between the subsystem designs may be necessary at the lower level. The first constraint represents those constraint conditions applicable directly to the subsystem design. The second constraint represents the requirement that the top level design variables applicable to subsystem I be the parameters held constant, or matched, during the subsystem optimization. The statement of the optimization problem for subsystem II is similar.

Gradients of the objective and constraints for the top level optimization are,

$$
\frac{d F}{d z}=\frac{\partial F}{\partial z}+\sum_{j} \frac{\partial F}{\partial q_{j}} \frac{\delta q_{j}^{*}}{\delta z}
$$

and

$$
\frac{d g_{i}}{d z}=\frac{\partial g_{i}}{\partial z}+\sum_{j} \frac{\partial g_{i}}{\partial q_{j}} \frac{\delta q_{j}^{*}}{\delta z}
$$

The $\delta / \delta z$ derivatives are pseudo-partial derivatives in that while they act as partial derivatives in equations 22 and 23 , they are constructed from sensitivities from the sublevels. The superscript asterisks indicate that optimum sensitivities from the subsystem designs are used,

$$
\frac{\delta q_{j}^{*}}{\delta z}=\frac{\partial q_{j}}{\partial z}+\sum_{k} \frac{\partial q_{j}^{*}}{\partial q_{z}} \sum_{i} \frac{\partial q_{k}}{\partial y_{1}} \frac{\partial y_{i}^{*}}{\partial z}
$$

The above equations are quite general and simplified in that no distinction is made between q's, y's, and $z$ 's for one subsystem and those for another. However, to do so would unnecessarily complicate the presentation of the equations and would not increase their generality. Application of these equations to any particular problem would involve careful attention to actual functional dependencies among the problem variables and parameters that would be unique to each application. The optimum sensitivities in the above equations come from differentiation of the necessary conditions of optimality, derived in reference 21 in a form that is typical of structural optimization problems. The essential information for computing the sensitivities of the optimal steady-state LQR problem is given briefly in this paper and in more detail in reference 22 . Accordingly, further analytical description of these sensitivities will not be given here and the interested reader is encouraged to consult references 21 and 22 .

\section{Present Formulation - Detailed}

The distinguishing element of the present formulation will be the decomposition of the structural subsystem to a sublevel equal to that of the control subsystem. Since the problem to be solved is the same as the benchmark example, the top level objective and constraints are similar to those in equations 10a-d, except that different top level design variables will be used. Also, the constraints are formulated as reciprocal Taylor series approximations. Since the benchmark example only uses structural design variables at the top level, the new top level design variables will only be structural as well. The structure is represented in the control design by its modal characteristics, so the top level design variables will be a subset of the squared natural frequencies of the open-loop structure. Specifically, the squared frequencies of modes $1,2,4,6,8,10$, and 12 will be used. In the following discussions, these will be denoted by $\omega_{\mathrm{p}}{ }^{2}$ 's, corresponding to the $z$ 's in the general formulation given previously. The remaining squared frequencies will be denoted by $\omega_{\mathrm{v}}{ }^{2}$ 's and will be treated as behavior variables, $\mathrm{q}_{\mathrm{s}}{ }^{\prime} \mathrm{s}$, from the structural subsystem.

The statement of the top level optimization problem is, then,

$$
\min _{\omega_{p}^{2}} \operatorname{Fr}=\operatorname{Tr}\left(G^{T} R_{G} G\right)
$$

subject to:

$$
\begin{gathered}
\mathrm{g}_{\mathrm{i}}=\overline{\mathrm{g}}_{\mathrm{O}_{\mathrm{i}}}+\sum_{\mathrm{h}} \frac{\mathrm{d} \overline{\mathrm{g}}_{\mathrm{i}}}{\mathrm{d} \omega_{\mathrm{h}_{\mathrm{h}}}^{2}}\left(\frac{\omega_{\mathrm{h}_{0}}^{2}}{\omega_{\mathrm{h}_{\mathrm{h}}}^{2}}\right)\left(\omega_{\mathrm{h}_{\mathrm{h}}}^{2}-\omega_{\mathrm{h}_{\mathrm{h}}}^{2}\right) \leq 0 \\
-\mu \omega_{\mathrm{p}_{\mathrm{o}}}^{2} \leq \omega_{\mathrm{h}_{\mathrm{h}}}^{2}-\omega_{\mathrm{p}_{\mathrm{h}_{0}}}^{2} \leq-\mu \omega_{\mathrm{p}_{\mathrm{h}}}^{2}
\end{gathered}
$$

where, for $\omega_{\mathrm{cl}_{1}}$.

$$
\overline{\mathbf{B}}=\left(\frac{\omega_{\mathrm{cl}_{1}}^{\mathbf{b}}-\omega_{\mathrm{cl}_{1}}}{\omega_{\mathrm{cl}_{1}}^{\mathbf{b}}}\right)
$$

The subscript 0 's denote starting values. The expressions for $\omega_{\mathrm{cl}_{2}}$ and $\xi_{\mathrm{cl}_{1}}$ are similar. Move limits of $\pm \mu$ of the current starting values at each step are imposed on the design variables as side constraints. 
The structural subsystem design is posed as an optimization problem to minimize weight with respect to the cross-sectional areas of the twelve truss members subject to the parameter matching constraints (i.e. equation 21c) and side constraints on the design variables. The authors did not wish to attempt enforcing strict equality for the parameter matching constraints, so the $\omega_{p}{ }^{2}$ 's desired from a top level design iteration were considered matched if the corresponding eigenvalues from the structural design were within a small tolerance, $\pm \varepsilon$. Thus the problem reads,

$$
\text { min } W(y)
$$

subject to, for each desired parameter, $\omega_{p}{ }^{2}$ :

$$
\left(\frac{1 \cdot\left(\frac{\omega_{p}^{2}(y)}{\omega_{p}^{2}}\right)}{\varepsilon}\right)^{2} \cdot 1 \leq 0
$$

and for each variable, $y$,

$$
10 \leq y \leq 2000
$$

There are no additional constraints (the $g_{i}$ 's of equation $21 \mathrm{~b}$ ) such as stress or deflection limits since there are none imposed in the benchmark example.

The LQR control subsystem design is the same as described by equations 5 through 8 . The real and imaginary parts of the closed-loop eigenvalues are behavior variables, $\mathrm{q}_{c}$, from the control subsystem. The expressions for the sensitivities are similar to equations 11 through 19 except that $y$ 's are replaced by $\omega_{\mathrm{p}}{ }^{2}$ 's for parameter sensitivities and by $\omega_{\mathrm{v}}{ }^{2} \mathrm{~s}$ for structural behavior variable sensitivities. Sensitivities of B are zero since it is assumed not a function of frequencies.

Once the subsystem optimizations are complete, optimum sensitivities with respect to parameters and behavior variables can be computed and the top level gradients can be constructed. Note that the physical coupling between the structure and control designs is one way, namely, from structure to control. Thus, none of the control behavior variables influences the structure design, whereas structural behavior variables (squared frequencies) do influence the control design. The first step is to calculate the pseudo-partial derivatives of behavior variables in equation 24 . For the structural behavior variables, $q_{j}=\omega_{\mathrm{Nj}}{ }^{2}$, equation 24 becomes,

$$
\frac{\delta \omega_{v_{j}}^{2}}{\delta \omega_{p}^{2}}=\sum_{1} \frac{\partial \omega_{v_{j}}^{2}}{\partial y_{1}} \frac{\partial y_{1}^{*}}{\partial \omega_{p}^{2}}
$$

The derivatives of the squared frequencies with respect to the structural design variables, $y$, are available from the implementation of the structural optimization step and are computed from equation 17. While they are not optimum sensitivities because they are not in any way computed from differentiation of the optimality conditions, they are the eigenvalue sensitivities for the structure at the optimum solution. For the control design behavior variables, equation 24 becomes (for the closed-loop eigenvalue imaginary parts only),

$$
\frac{\delta \omega_{c_{j}}^{*}}{\delta \omega_{p}^{2}}=\frac{\partial \omega_{c_{j}}^{*}}{\partial \omega_{p}^{2}}+\sum_{k} \frac{\partial \omega_{c_{j}}^{*}}{\partial \omega_{v_{k}}^{2}} \sum_{i} \frac{\partial \omega_{\mathrm{N}_{k}}^{2}}{\partial y_{1}} \frac{\partial y_{1}^{*}}{\partial \omega_{p}^{2}}
$$

Note that the last summation in equation 28 is simply the expression in equation 27 . The expression for the eigenvalue real parts is similar.

The gradients for the top level objective and constraint functions can now be obtained, with reference to equations 27 and 28 , as

$$
\begin{gathered}
\frac{d F_{T}}{d \omega_{p}^{2}}=\left(\frac{\partial G^{T}}{\partial \omega_{p}^{2}} R_{G} G+G^{T} R_{G} \frac{\partial G^{*}}{\partial \omega_{p}^{2}}\right) \\
+\sum_{j}\left(\frac{\partial G^{T}}{\partial \omega_{N_{j}}^{2}} R_{G} G+G^{T} R_{G} \frac{\partial G^{*}}{\partial \omega_{N_{j}}^{2}}\right) \frac{\delta \omega_{N_{j}}^{2}}{\delta \omega_{p}^{2}} \\
\frac{d g_{i}}{d \omega_{p}^{2}}=\frac{d \bar{g}_{i}^{*}}{d \omega_{p}^{2}}\left(\frac{\omega_{p_{0}}^{2}}{\omega_{p}^{2}}\right)^{2}
\end{gathered}
$$

where, for $\omega_{\mathrm{cl}_{1}}$,

$$
\frac{d \bar{g}\left(\omega_{c_{1}}\right)^{*}}{d \omega_{p}^{2}}=\frac{\partial \tilde{g}\left(\omega_{\mathrm{cl}_{l}}\right)}{\partial \omega_{\mathrm{cl}_{1}}} \frac{\delta \omega_{\mathrm{cl}_{1}}^{*}}{\delta \omega_{\mathrm{p}}^{2}}
$$

\section{Implementation}

The top level and both sublevel optimizations were written as separate codes that communicated through data files. The ADS [26] optimization code was used at the top level and structural subsystem while the optimum LQR solution was obtained using the ORACLS optimal control design software package [27]. The Sequential Quadratic Programming strategy was used at the top level with the Modified Method of Feasible Directions optimizer and the one dimensional 
search option wherein the minimum of the constrained function is found by first finding the bounds and then using polynomial interpolation. The same options were used in the structural subsystem optimization. The move limit, $\mu$, on the lop level design variables was set initially to $5 \%$. This was reduced as the final solution was approached to overcome oscillation in the constraints. The tolerance, $\varepsilon$, in the parameter matching constraints was set to $1 \%$.

The structural subsystem optimizations tended to experience difficulty satisfying the parameter matching constraints (equation 26b) since any top level design variable change of more than $1 \%$ resulted in an infeasible starting point at the structure sublevel. Thus, an intermediate step was introduced into the procedure by which the structural design variables were adjusted until the $1 \%$ parameter matching constraints were met. These values of the structural design variables then served as the starting point for the structural subsystem optimization. The adjustments were automated by forming an optimization problem (again, using ADS) wherein a cumulative function of the parameter matching constraints is minimized with respect to the structural design variables until the function becomes negative, thus ensuring that the constraints were satisfied. A cumulative function with this desirable property is the so-called KS function [28]. For a set of NC constraints, $g_{i}$, the function is ,

$$
K S=\frac{1}{\rho} \ln \sum_{i=1}^{X C} e^{\rho g_{i}}
$$

where $\rho$ is a weighting parameter selected so as not to produce an over-or underflow condition when computing exponentials of large positive or negative numbers. This function has the property that it is always greater than or equal to the maximum constraint within a definite band,

$$
8_{i_{\max }} \leq K S \leq 8_{i_{\max }}+\frac{1}{\rho} \ln (\mathrm{NC})
$$

The algorithm proceeds as follows. The matching tolerance is set to $4 \%$ (one percentage point less than the top level move limit) and the first matching constraint is put into the cumulative function which is reduced by the optimizer until it becomes negative. Then the first matching constraint serves additionally as an actual constraint while the second matching constraint is added to the cumulative function which, again, is reduced by the optimizer until it becomes negative. This continues until all of the matching constraints (seven of them, in this case) satisfy the $4 \%$ tolerance. The tolerance is then reduced to $3 \%$ and the process starts again with the first matching constraint. This continues until all of the matching constraints satisfy the $1 \%$ tolerance.

\section{NUMERICAL RESULTS AND DISCUSSION}

Figures 4 and 5 show the line-of-sight (LOS) error responses (total lateral deflection of node 1 away from the $z$ axis) of the open- and closed-loop initial structure to a unit displacement of node 2 in the $x$ direction imposed as an initial condition. The control manages to suppress the large excursions, but reduces the average deflections little. Also, the damping appears light to moderate. In contrast, figure 6 shows the response of the present design. The overall response is drastically reduced as are the larger excursions, and the system is more highly damped. Note the expanded scale of figure 6.

Structural member sizes, and open- and closedloop eigenvalues for the present design are shown in Tables III through $\mathrm{V}$ along with corresponding results for 'Design II' and 'Design III' from reference 17 and the initial design. It can be seen in Table IV and figure 7 that the present design is an improvement over 'Designs II and III' in not only the Frobenius gain norm, which was the objective function in all cases, but also in the weight of the structure, which was the objective function in the present method's structural subsystem design. Whereas, in the benchmark examples, the member sizes were allowed to range widely so as to satisfy the problem constraints as easily as possible, they were rather more constrained in the present example to produce the desired open-loop frequencies always at a minimum weight. This demand is undoubtedly responsible for the reduced weight of the present design. Since the structural mass is assumed negligible in the dynamics, it is consistent with the reduced weight that both the open- and closed-loop frequencies in general show the present design to be a more flexible one.

Several attempts were made to reduce the degree to which the physical coupling between the subsystems was accommodated. One approach was to pass the modal information resulting from the parameter matching program to the control design. The other was to pass the desired frequencies from the top level and the mode shapes from the previous structural design. Both approaches failed to converge to a feasible design. It is believed that the sensitivities from the subsystem designs used at the top level were inconsistent with one another resulting in erroneous top level gradients. 


\section{CONCLUSIONS}

A method for integrated control/structure optimum design by multilevel decomposition has been presented. It was applied to a design problem found in the literature and the results were compared. The method was found to produce lower values of the objective function, which was the Frobenius norm of the state feedback gain matrix, as well as the structural weight. It is concluded that using structural weight as the decomposed structural subsystem design objective has a moderating influence on the weight of the design. It was also found that the physical coupling between the subsystems must be considered in the design procedure. The structure-to-control coupling that is present in this example will occur whenever the control synthesis is model-based.

\section{ACKNOWLEDGMENT}

The authors wish to acknowledge Mr. Benjamin B. James with whom many fruitful discussions of multilevel decomposition were held.

\section{REFERENCES}

1. Junkins, J. L.; and Rew, D. W.: Unified Optimization of Strucrures and Controllers. Large Space Structures: Dynamics and Control. Atluri. S. N., and Amos, A. K., editors, Springer-Verlag 1988.

2. Hale, Arthur L.; and Lisowski, Ronald J.: Optimal Simultaneous Structural and Control Design of Maneuvering Flexible Spacecraft. Proceedings of the 4th VPI\&SUIAIAA Symposium on Dynamics and Conirol of Large Space Siructures, Blacksburg, Va.. June 6-8 1983.

3. Hale, A. L.; Lisowski, R. J.; and Dahl, W. E.: Optimizing Both the Structure and Control of Maneuvering Flexible Spacecraft. AAS/AIAA Astrodynamics Conference, August 1983.

4. Lisowski, Ronald J.; and Hale, Arthur L.: Optimal Design for Single Axis Rotational Maneuvers of a Flexible Structure. AIAA Paper No. 84-1041, 25th SDM Proceedings, Palm Springs, Califomia, May 14161984.

5. Salama, M.; Hamidi, M.; and Demsetz, L.: Optimization of Controlled Structures. Jet Propulsion Workshop on Identification and Control of Flexible Space Structures, San Diego, Califomia, 1984.

6. Hale, Arthur L.: Integrated Structural/Control Synthesis via Set-Theoretic Methods. AIAA Paper No. 85-0806.

7. Haftka, Raphael, T.; Martinovic, Zoran N.; and Hallauer. William L. Jr.: Enhanced Vibration Controllability by Minor Structural Modifications.
A/AA Journal, Vol. 23, No. 8. August 1985, pp. 1260 $-1266$.

8. Bodden, D. S.; and Junkins, J. L.: Eigenvalue Optimization Algorithms for Structure/Controller Design Iterations. AJAA Journal of Guidance and Control, Vol. 8, No. 6, Nov.-Dec. 1985.

9. Onoda, Junjiro; and Haftka, Raphael T.: Simultaneous Structure/Control Optimization of Large Flexible Spacectaft ALAA Paper No. 87-0823.

10. Miller, David F; and Shim, Jaedong: GradientBased Combined Structural and Control Optimization. AIAA Journal of Guidance. Control, and Dynamics, Vol. 10. No.3, May-June 1987.

11. Lim, Kyong B.; and Junkins, John L.: Robustness Optimization of Structural and Controller Parameters. ALAA Paper No. 87-0791.

12. Lust, R. V.; and Schmit, L. A.: ControlAugmented Structural Synthesis. AlAA Journal, Vol. 26. No. 1. January 1988.

13. Zeiler, T. A.; and Weisshaar, T. A.: Integrated Aeroservoelastic Tailoring of Lifting Surfaces. AIAA Jownal of Aircraft, Vol. 25 . No. 1, January 1988.

14. Belvin. W. Keith; and Park, K. C.: Structural Tailoring and Feedback Control Synthesis: An Interdisciplinary Approach. AIAA Paper No. 88 2206, 29th SDM Proceedings, Williamsburg. Va., April $1 \overline{8}-\overline{2} \overline{0} \quad 19 \overline{8} \overline{8}$.

15. Salama, M.; Garba, J.; and Demsetz, L.: Simultaneous Optimization of Controlled Structures. Computational Mechanics 3 (1988), pp. 275-282.

16. Gilbert, Michael. G.; and Schmidt, David K.: Integrated Structure/Control Law Design by Multilevel Optimization. AIAA Paper No. 89-3470, ALAA Guidance, Navigation and Control Conference, Boston, MA, Augusi 14-16 1989.

17. Grandhi, Ramana V:S Structural and Control Optimization of Space Structures. Computers and Siructures, Vol. 31, No. 2, 1989, pp. 139-150.

18. Thomas, H. L.; and Schmit, L. A. Jr.: Control Augmented Suructural Synthesis With Dynamic Stability Constraints. AIAA Paper No. 89-1216, 30th SDM Proceedings, Mobile, Alabama, April 3-5 1989.

19. Onoda, Junjiro; and Watanabe, Naoyuki: Integrated Direct Optimization of Structure / Regulator / Observer for Large Flexible Spacecraft. AIAA Paper No. 89-1313. 30th SDM Proceedings. Mobile. Alabama, April 3-5 1989.

20. Sobieszczanski-Sobieski, J.; A Linear Decomposition Method for Large Optimization Problems - A Blueprint for Development. NASA TM83248, Feb. 1982. 
21. Sobieszczanski-Sobieski, J.; Barthelemy, J.; and Riley, K.: Sensitivity of Optimum Solutions to Problem Parameters. AJAA Journal, Vol. 20, No. 9 , Sept. 1982, pp. 1291-1299.

22. Gilbert, M. G.: Design Parameter Sensitivity Methods in Optimal LQG Control Law Synthesis. AlAA Paper No. 85-1930, August 1985.

23. Sobieszczanski-Sobieski, J.; James, B. B.: and Dovi, A. R.: Structural Optimization by Multi-

level Decomposition. AIAA Journal. Vol. 23, No. 11. Nov. 1985, pp. 1775-1782.

24. Sobieszczanski-Sobieski, J.; James, B. B.; and Riley. M. F.: Structural Sizing by Generalized, Multilevel Optimization. AIAA Journal, Vol. 25. No. 1. January 1987, p. 139.

25. Charles Stark Draper Laboratory. Inc.: "ACOSS Four (Active Control of Space Structures), Theory," Vols. I and II (Appendix), RADC-TR-80-78. April 1980.

26. Vanderplaats, G. N.: "ADS - A Fortran Program for Automated Design Synthesis - Version 1.10," NASA CR-177985, September 1985.

27. Armstrong, E. A.: "ORACLS - A System for Linear-Quadratic-Gaussian Control Law Design," NASA TP 1106, 1978.

28. Kreisselmeier, G.; Steinhauser, R.: "Systematic Control Design by Optimizing a Vector Performance Index." Computer Aided Design of Control Systems, M. A. Cuemod, Editor, Pergamon Press, Inc.

Table I ACOSS -4 Nodal Coordinates

\begin{tabular}{|c|c|c|c|}
\hline Vode & $x$ & $y$ & $z$ \\
\hline 1 & 0.0 & 0.0 & 10.165 \\
2 & -5.0 & -2.887 & 2.0 \\
3 & 5.0 & -2.887 & 2.0 \\
4 & 0.0 & 5.7735 & 2.0 \\
5 & -6.0 & -1.1547 & 0.0 \\
6 & -4.0 & -4.6188 & 0.0 \\
7 & 4.0 & -4.6188 & 0.0 \\
8 & 6.0 & -1.1547 & 0.0 \\
9 & -2.0 & 5.7735 & 0.0 \\
10 & 2.0 & 5.7735 & 0.0 \\
\hline
\end{tabular}

Table II ACOSS-4 Elements

\begin{tabular}{|c|c|c|}
\hline Element & $\begin{array}{c}\text { Nodal } \\
\text { Connectivities }\end{array}$ & $\begin{array}{c}\text { Cross Sectional } \\
\text { Area }\end{array}$ \\
\hline 1 & $1 \cdot 2$ & 1000 \\
2 & $2 \cdot 3$ & 1000 \\
3 & $2 \cdot 4$ & 1000 \\
4 & $3 \cdot 4$ & 1000 \\
5 & $1 \cdot 3$ & 100 \\
6 & $1 \cdot 4$ & 100 \\
7 & $2 \cdot 5$ & 100 \\
8 & $2 \cdot 6$ & 100 \\
9 & $3 \cdot 7$ & 100 \\
10 & $3 \cdot 8$ & 100 \\
11 & 4.9 & 100 \\
12 & 4.10 & 100 \\
\hline
\end{tabular}

Table III ACOSS-t Member CrossSectional Areas

\begin{tabular}{|c|c|c|c|c|}
\hline $\begin{array}{c}\text { Member } \\
\text { No. }\end{array}$ & $\begin{array}{l}\text { Initlal } \\
\text { Design }\end{array}$ & $\begin{array}{l}\text { - Desigh a } \\
\text { (Rel. I7) }\end{array}$ & $\begin{array}{c}\text { 'Desiza III' } \\
\text { (Rof. 17) }\end{array}$ & $\begin{array}{l}\text { Prescant } \\
\text { Desige }\end{array}$ \\
\hline 1 & 1060 & 998.07 & 175.28 & 1040.16 \\
\hline 2 & 1000 & 998.07 & 1991.30 & 1014.46 \\
\hline $\mathbf{3}$ & 1000 & 998.07 & 1351.59 & 1054.62 \\
\hline 4 & 1000 & 998.06 & 1034.27 & 1023.21 \\
\hline 5 & 100 & 996.58 & $1 \% 2.14$ & 171.63 \\
\hline 6 & 160 & 998.10 & 1985.75 & 242.75 \\
\hline 7 & 100 & 921.64 & 162.38 & 140.11 \\
\hline $\mathbf{s}$ & 100 & 982.75 & 159.91 & 153.71 \\
\hline , & 100 & 36.93 & 29.20 & 96.99 \\
\hline 10 & 100 & 194.24 & 15286 & 217.97 \\
\hline 11 & 100 & 30.09 & 29.19 & 29.06 \\
\hline 12 & 100 & 67.51 & 467.09 & 201.62 \\
\hline $\begin{array}{l}\text { Frobenius } \\
\text { Norm }\end{array}$ & 17.175 & 21.969 & 18.431 & 17.945 \\
\hline Weight & 40.69 & 66.036 & 97.134 & 47,843 \\
\hline
\end{tabular}

Table IV ACOSS-4 Structural Elgenvalues (Squared Frequencies)

\begin{tabular}{|c|c|c|c|c|}
\hline $\begin{array}{c}\text { Mode } \\
\text { No. }\end{array}$ & $\begin{array}{c}\text { Inilial } \\
\text { Design }\end{array}$ & $\begin{array}{c}\text { 'Design } \\
\text { (Ref. 17) }\end{array}$ & $\begin{array}{c}\text { 'Design In' } \\
\text { (Ref. 17) }\end{array}$ & $\begin{array}{c}\text { Present } \\
\text { Design }\end{array}$ \\
\hline 1 & 1.80 & 2.08 & 1.89 & 1.81 \\
2 & 2.77 & 3.13 & 2.78 & 4.72 \\
3 & 8.35 & 5.57 & 4.54 & 5.71 \\
4 & 8.74 & 15.91 & 15.31 & 13.88 \\
5 & 11.54 & 33.49 & 17.60 & 17.72 \\
6 & 17.67 & 39.31 & 34.95 & 18.58 \\
7 & 21.73 & 85.79 & 42.95 & 38.83 \\
8 & 22.61 & 9451 & 96.37 & 39.49 \\
9 & 72.92 & 101.50 & 112.70 & 81.19 \\
10 & 85.57 & 177.30 & 172.30 & 94.89 \\
11 & 105.77 & 202.70 & 214.60 & 111.98 \\
12 & 166.54 & 231.30 & 310.90 & 17475 \\
\hline
\end{tabular}


Table V ACOSS-4 Closed-Loop Eigenvalues

\begin{tabular}{|c|c|c|c|}
\hline $\begin{array}{c}\text { Initial } \\
\text { Design }\end{array}$ & $\begin{array}{c}\text { 'Design II' } \\
\text { (Ref. 17) }\end{array}$ & $\begin{array}{c}\text { 'Design III' } \\
\text { (Ref. 17) }\end{array}$ & $\begin{array}{c}\text { Present } \\
\text { Design }\end{array}$ \\
\hline \hline $0.073 \pm 1.342 \mathrm{j}$ & $-0.246 \pm 1.435 \mathrm{j}$ & $-0.252 \pm 1.369 \mathrm{j}$ & $-0.207 \pm 1.342 \mathrm{j}$ \\
$-0.109 \pm 1.663 \mathrm{j}$ & $-0.256 \pm 1.762 \mathrm{j}$ & $-0.251 \pm 1.670 \mathrm{j}$ & $-0.126 \pm 2.178 \mathrm{j}$ \\
$-0.213 \pm 2.885 \mathrm{j}$ & $-0.274 \pm 2.346 \mathrm{j}$ & $-0.185 \pm 2.113 \mathrm{j}$ & $-0.232 \pm 2.372 \mathrm{j}$ \\
$0.237 \pm 2.950 \mathrm{j}$ & $-0.266 \pm 3.980 \mathrm{j}$ & $-0.267 \pm 3.905 \mathrm{j}$ & $-0.220 \pm 3.720 \mathrm{j}$ \\
$-0.285 \pm 3.388 \mathrm{j}$ & $-0.275 \pm 5.781 \mathrm{j}$ & $-0.295 \pm 4.185 \mathrm{j}$ & $-0.262 \pm 4.202 \mathrm{j}$ \\
$-0.363 \pm 4.191 \mathrm{j}$ & $-0.231 \pm 6.265 \mathrm{j}$ & $-0.344 \pm 5.903 \mathrm{j}$ & $-0.337 \pm 4.298 \mathrm{j}$ \\
$-0.355 \pm 4.649 \mathrm{j}$ & $-0.236 \pm 9.259 \mathrm{j}$ & $-0.265 \pm 6.548 \mathrm{j}$ & $-0.354 \pm 6.222 \mathrm{j}$ \\
$-0.344 \pm 4.744 \mathrm{j}$ & $-0.261 \pm 9.718 \mathrm{j}$ & $-0.309 \pm 9.812 \mathrm{j}$ & $-0.302 \pm 6.239 \mathrm{j}$ \\
$-0.292 \pm 8.535 \mathrm{j}$ & $-0.180 \pm 10.075 \mathrm{j}$ & $-0.290 \pm 10.613 \mathrm{j}$ & $-0.300 \pm 9.005 \mathrm{j}$ \\
$-0.276 \pm 9.247 \mathrm{j}$ & $0.217 \pm 13.314 \mathrm{j}$ & $-0.292 \pm 10.004 \mathrm{j}$ & $-0.284 \pm 9.737 \mathrm{j}$ \\
$-0.214 \pm 10.283 \mathrm{j}$ & $-0.339 \pm 14.234 \mathrm{j}$ & $-0.231 \pm 10.777 \mathrm{j}$ & $-0.222 \pm 10.58 \mathrm{j}$ \\
$-0.083 \pm 12.905 \mathrm{j}$ & $-0.284 \pm 15.200 \mathrm{j}$ & $-0.092 \pm 13.323 \mathrm{j}$ & $-0.091 \pm 13.219 \mathrm{j}$ \\
\hline
\end{tabular}

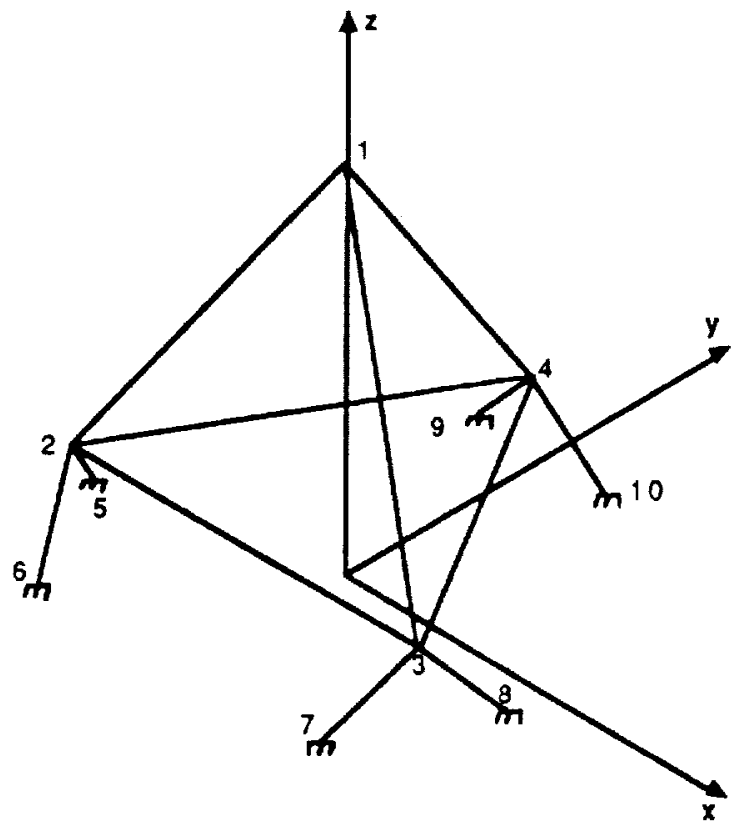

Figure 1 ACOSS-4 Structural Model

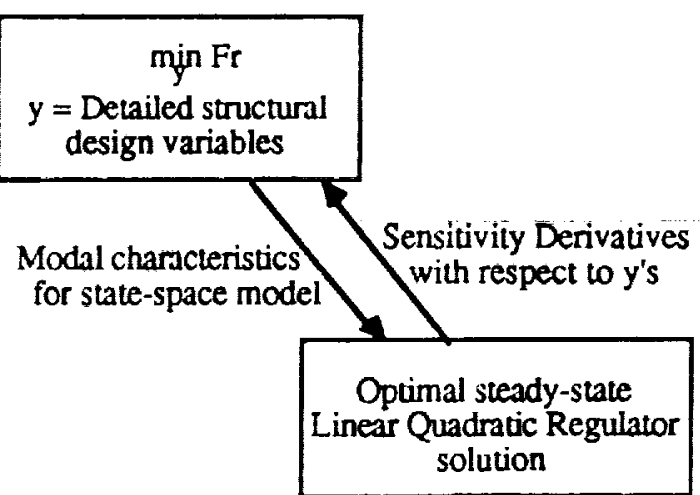

Figure 2 Partial Decomposition of Controlled Structure 


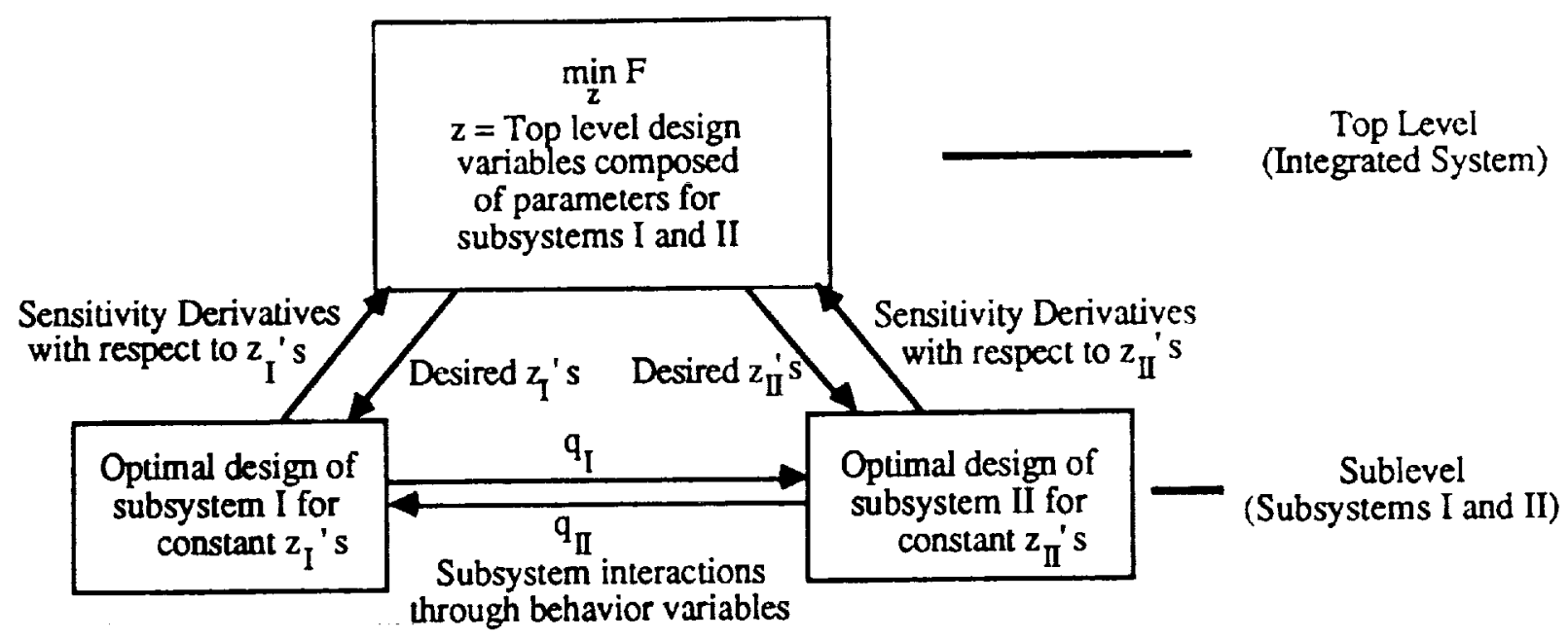

Figure 3 Generalized Decomposition

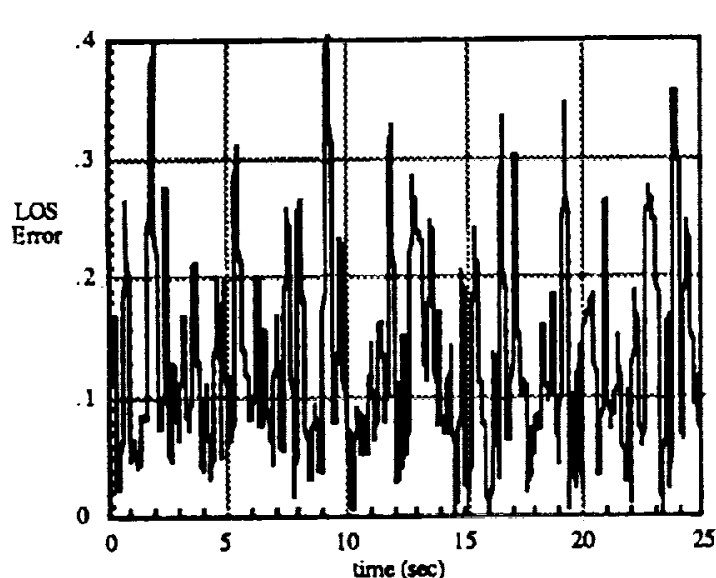

Figure + Open-Loop Response of Initial Structure

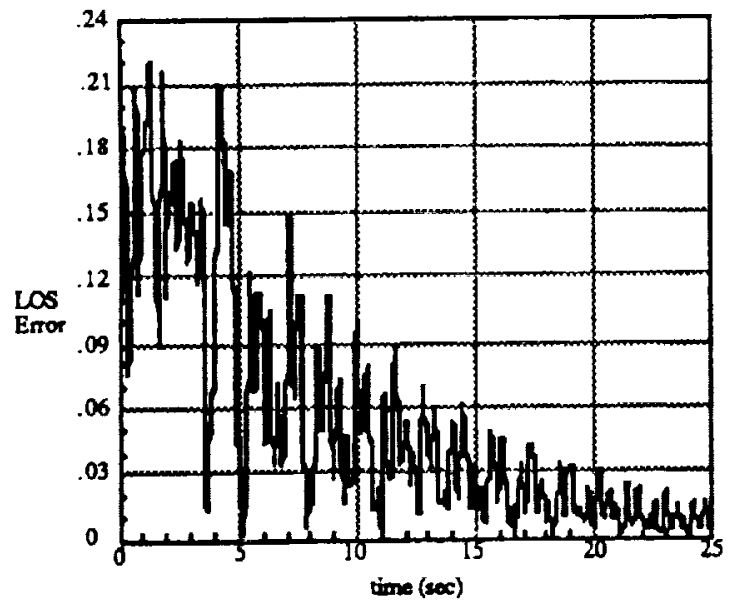

Figure 6 Closed-Loop Response of Present Design

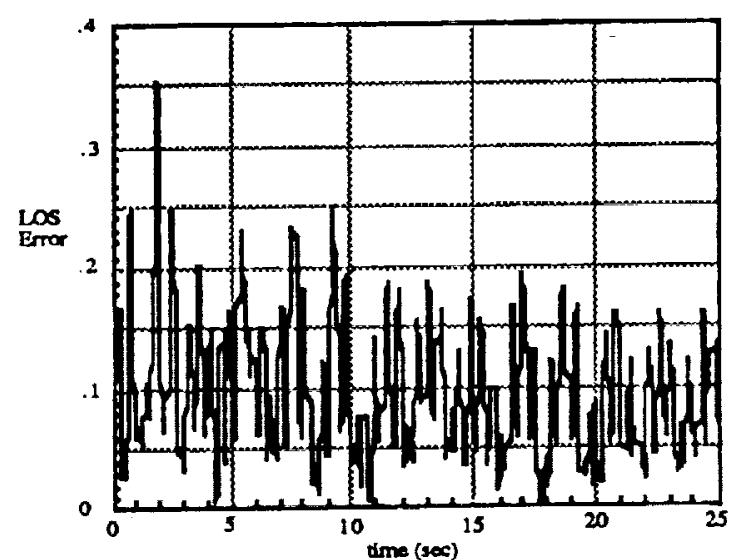

Figure 5 Closed-Loop Response of Initial Structure

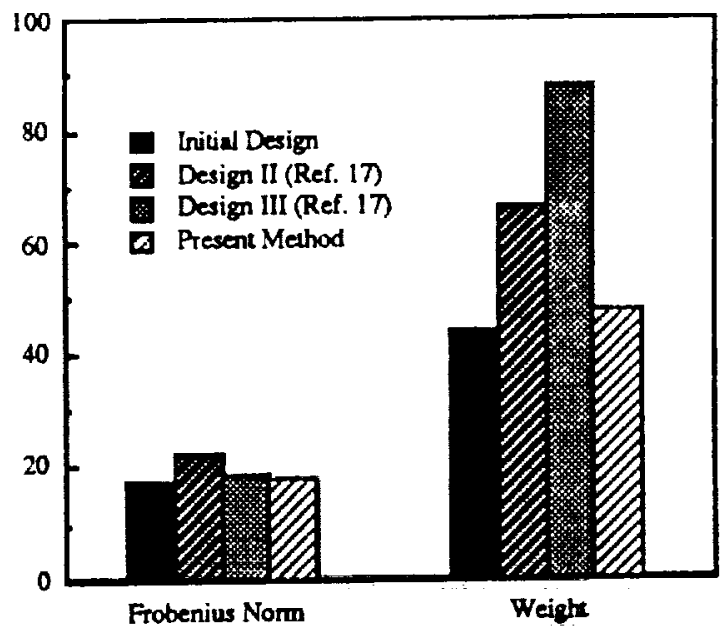

Figure 7 Comparison of Results for Frobenius Gain Norm and Structural Weight 


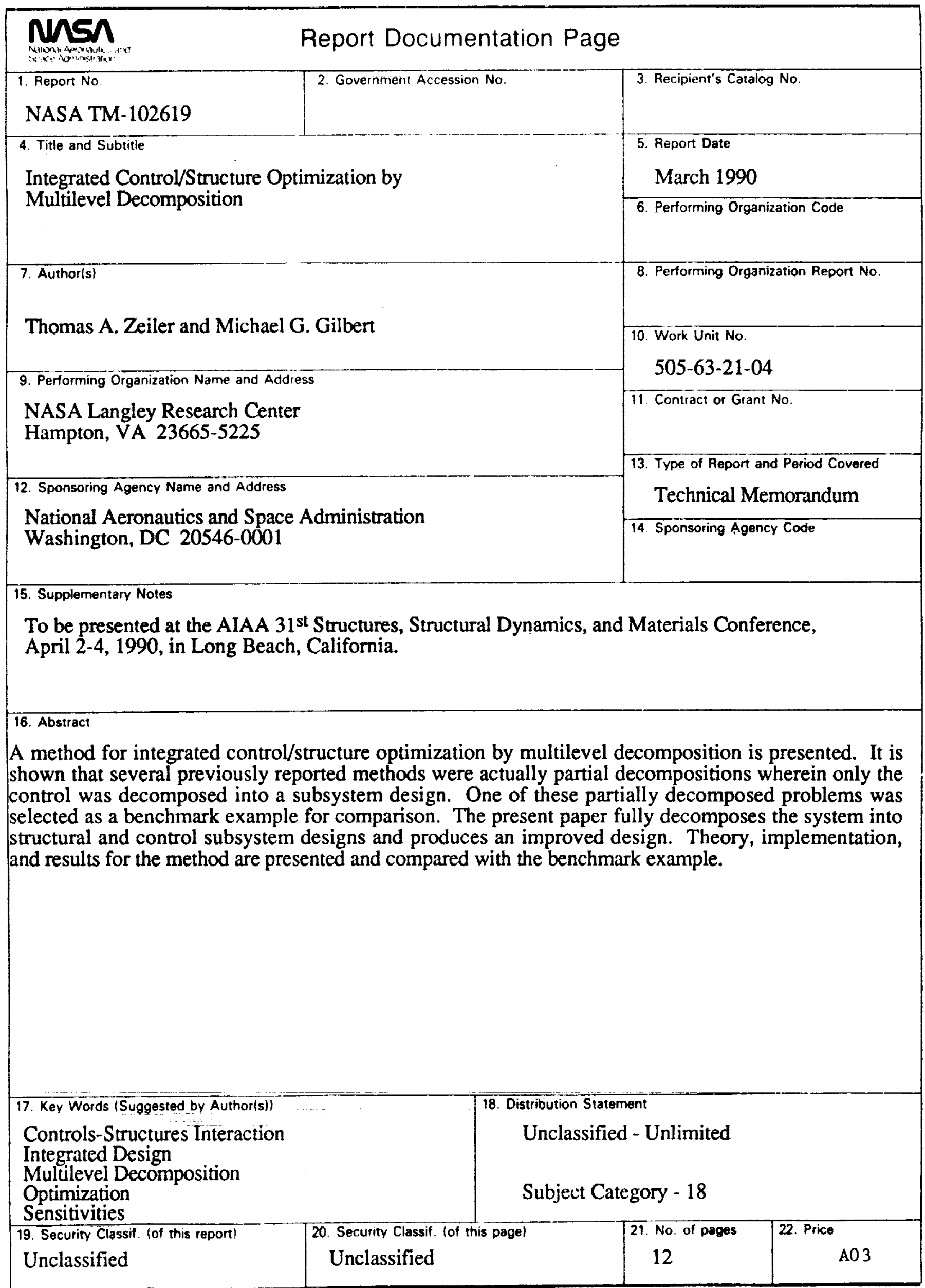

